\title{
DOUBLE TRANSABDOMINAL IMPALEMENT INJURY WITHOUT MAJOR VASCULAR AND VISCERAL TRAUMA: A SUI GENERIS
}

\author{
Darwin Britto Devadhason1, Tharali Chandrasekar Narendran², Alankrith Ramesh Kashyap³, Raveendran Rani Arun Prasanth4, \\ Yella Suryakiran 5
}

\begin{abstract}
${ }^{1}$ Assistant Professor, Department of General Surgery, SRM Medical College, Hospital \& Research Centre, Chennai. ${ }^{2}$ Assistant Professor, Department of General Surgery, SRM Medical College, Hospital \& Research Centre, Chennai. ${ }^{3}$ Post Graduate, Department of General Surgery, SRM Medical College, Hospital \& Research Centre, Chennai. ${ }^{4}$ Post Graduate, Department of General Surgery, SRM Medical College, Hospital \& Research Centre, Chennai. 5 Post Graduate, Department of General Surgery, SRM Medical College, Hospital \& Research Centre, Chennai.
\end{abstract}

\begin{abstract}
Impalement injury is one of the most gruesome forms of injury that can occur and death of the individual at the site of happenstance is almost certain due to trauma to major visceral and vascular structures and if at all he does manage to survive to be brought to an emergency room, he incurs the storm of uncontrolled haemorrhage, severe sepsis, metabolic and haemodynamic disturbances which pose a considerable threat for intra-operative and post-operative wellbeing. A timely, systematic, prudent multimodality can be of great assistance for patient survival as is presented in this case of impalement abdominal injury in the right iliac fossa region.
\end{abstract}

\section{KEYWORDS}

Double Impalement, Transabdominal Trauma, Absent Visceral and Vascular Injury.

HOW TO CITE THIS ARTICLE: Devadhason DB, Narendran TC, Kashyap AR, et al. Double transabdominal impalement injury without major vascular and visceral trauma: a sui generis. J. Evolution Med. Dent. Sci. 2016;5(41):2563-2565,

DOI: $10.14260 /$ jemds/2016/599

\section{INTRODUCTION}

Impalement injury, a form of penetrating injury abdomen occurs due to fall on a sharp object which can be lifethreatening, as they can cause serious visceral and vascular trauma, but incidence of projecting in-situ rods is very rare. $[1,2,3]$ Though limited cases have been reported, the patient survival remains dubious as death at the site is de facto; if at all they are fortunate to survive, they not only pose challenges for proper management to the surgical and anaesthetic personnel but also suffer from long-term morbidity. ${ }^{[4]}$ We would like to present a unique case of impalement injury by two parallel rusted iron rods to the right iliac fossa very close to major viscera and vasculature, yet not causing any damage to these structures and patient recovery being uneventful - a near escape prospect.

\section{CASE REPORT}

A 23-year-old boy came with an alleged history of fall from a height into two sharp rusted iron rods at his construction site workplace. He was brought half an hour late from the time of his injury as the rods had to be cut away from the underlying attached concrete slabs. He was brought by an emergency team of paramedics with both the rods impaled into his right lower abdomen. His hip was in a flexion and caused him severe pain on attempts of passive extension [Figure 1].

Financial or Other, Competing Interest: None.

Submission 06-04-2016, Peer Review 02-05-2016,

Acceptance 07-05-2016, Published 23-05-2016.

Corresponding Author:

Dr. Alankrith Ramesh Kashyap,

Room 303,

PG Medical Gent's Hostel,

SRM College Hospital \& Research Centre,

SRM Nagar

Kattankulathur, Chennai-603203.

E-mail: koolchant@gmail.com

DOI: 10.14260/jemds/2016/599

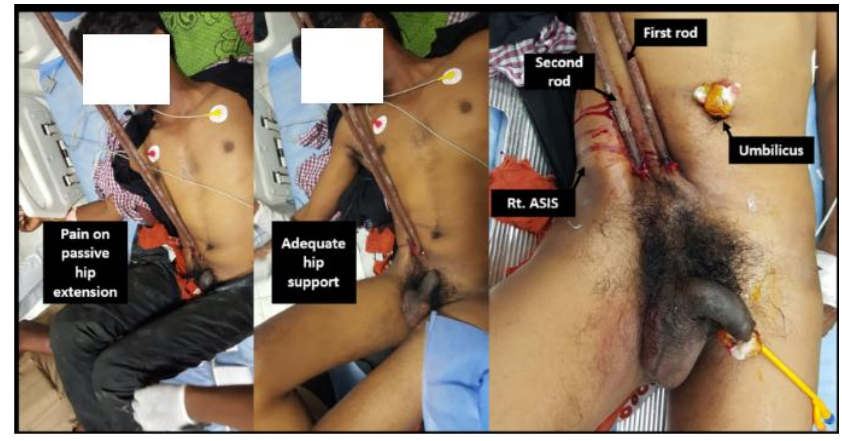

Fig. 1

He had no loss of conscious, seizures, vomiting after the fall; though he did suffer from minor lacerations on his thighs and scrotum with multiple abrasions over his body. He had an urge to pass urine, but could not do so since the time of fall. There was no history of bleeding per urethra. On careful and successive examination by our team, he continued to have persistent tachycardia (Pulse $>98 /$ minute), but blood pressure remained normal. He was febrile $\left(100^{\prime} \mathrm{F}\right)$. He was maintaining saturation more than $95 \%$ at room air. Per abdomen examination revealed guarding but no rigidity and there was no evidence of free fluid within peritoneal cavity.

His knees and hip were given adequate support to maintain them in flexed position and an urgent FAST, though suboptimal was done, which revealed no significant damage to solid organs, minimal free fluid. Aorta was normal up to the bifurcation of common iliac arteries beyond which could not be visualized due to artifacts and uncooperative nature of the patient. The patient was promptly catheterized-clear urine drained, emergency blood investigations sent, adequate blood reservation, tetanus toxoid shot given. His total count was 27000 cells/cu.mm (Normal $4000-11000$ cells/cu.mm). Due to the uncooperative nature of the patient and difficulty in 
positioning, a pre-operative CT (Computed Tomography) could not be done.

A provisional diagnosis of traumatic small bowel perforation was made and a high risk consent was obtained for exploratory laparotomy and the patient was put out under general anaesthesia. The patient was shifted to the operation with the unmanipulated rods in situ.

\section{Intra-Operative Findings}

A midline incision was made and abdomen explored. Intraoperative findings revealed two parallel rusted iron rods, first extending from the skin to just $10 \mathrm{~cm}$ proximal to right Common Iliac Artery (CIA) causing a small mesenteric tear in the jejunum $80 \mathrm{~cm}$ distal to DJ flexure with minimal grazing of the seromuscular layer of the jejunum and second from skin through the retroperitoneum with its tip $30 \mathrm{~cm}$ from the bladder [Figure 2, 3, 4].

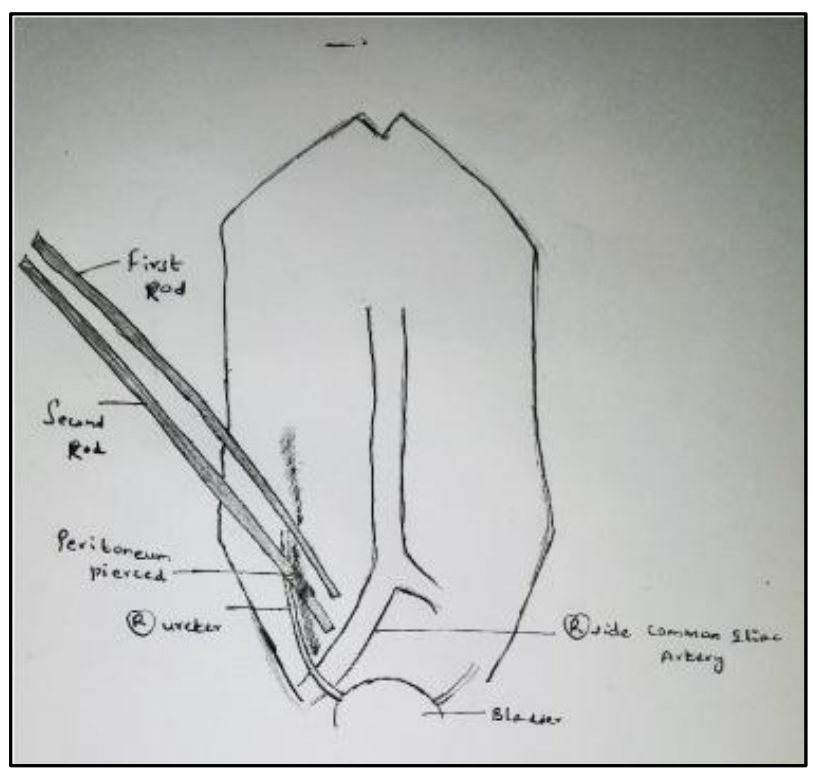

Fig. 2

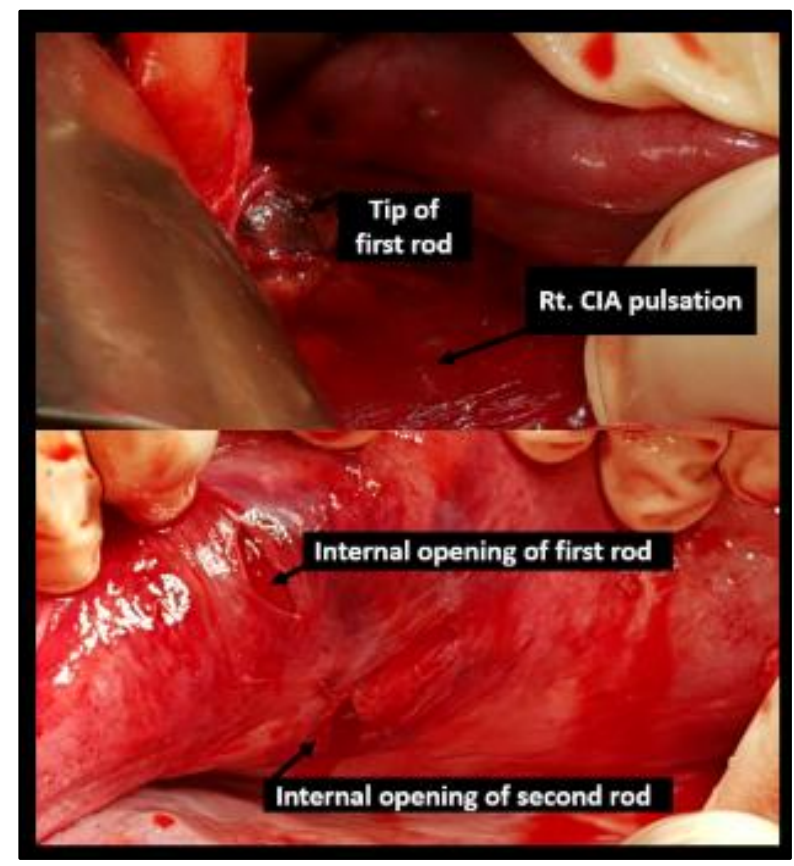

Fig. 3
The grazed bowel segment was hyperaemic but viable with few omental tears [Figure 4].

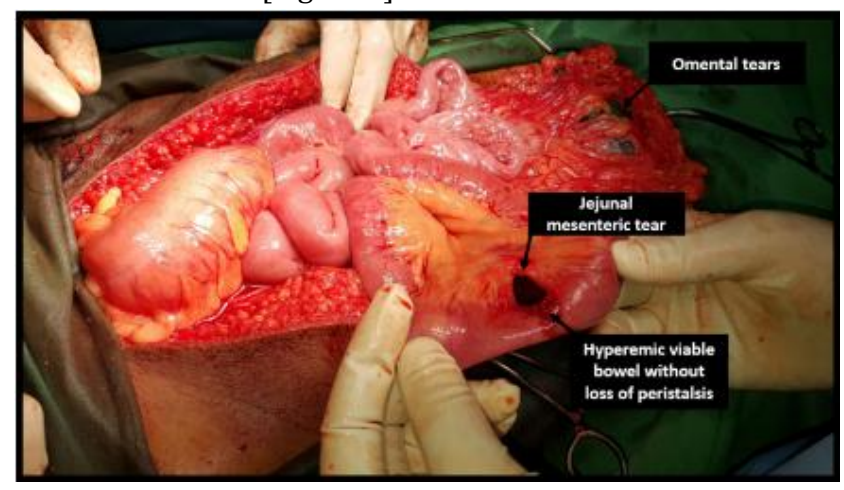

Fig. 4

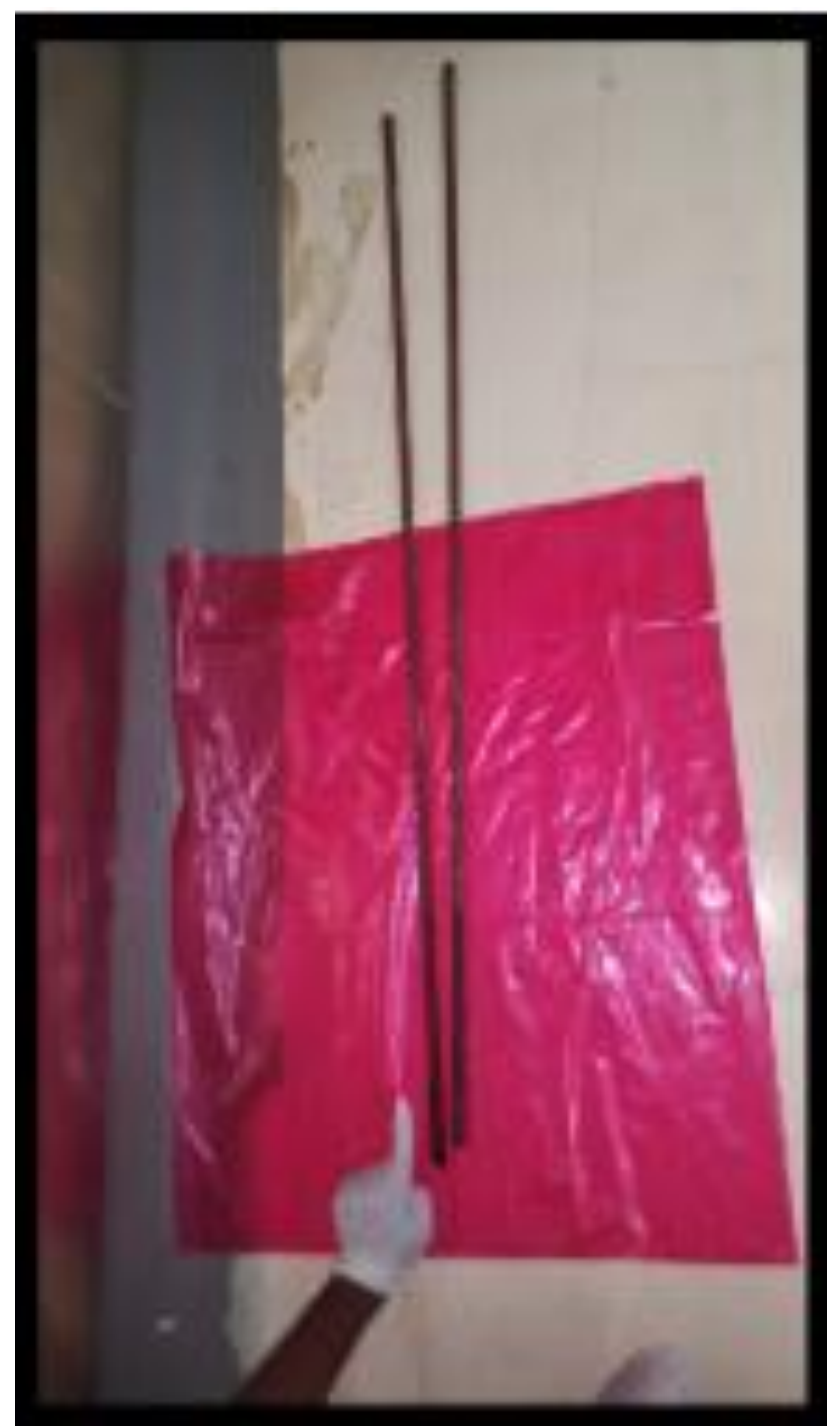

Fig. 5

The right ureter was found to be narrowly missed when the retroperitoneum was opened to remove the second rod. Resection and anastomosis of the hyperaemic bowel segment was done to prevent any sepsis due to the rusted rod and a thorough peritoneal lavage was given. Drain was kept in the pelvis and abdomen closed. Patient was started on a cocktail of Amikacin, Cefoperazone-Sulbactam and Metronidazole therapy. Patient was kept under thorough observation. 


\section{Post-Operative Status}

Post-operatively, patient was shifted for a CT Brain, Chest and Abdomen. CT Brain revealed no abnormality. CT Chest and Abdomen revealed only non-significant post-operative changes.

Patient improved drastically after the procedure without any of deterioration and made an uneventful post-operative recovery.

\section{DISCUSSION}

Penetrating wounds of abdomen account for $25 \%$ of all abdominal trauma, which include stab and gunshot wounds; and evisceration of bowel or omentum is associated with an injury rate of $78 \%$, thus warrants a laparotomy.[2,5] Determining the trajectory of the impaled object during physical examination can aid in a rough estimation of the structures that could have been injured with small bowel being the most common organ to be damaged 95\%. ${ }^{[6]}$ Once a penetrating abdominal trauma to vital structures is suspected, no time should be wasted in obtaining image based studies, particularly in scenarios where patient has persistent tachycardia, hypotension, signs of peritonitis, retained impaled foreign body; and should be straight away shifted to operation theatre; the impaled structure acts like a tamponade over important vascular structures.[7] The traversed organs should be visualized directly and only then attempts of removal of the foreign body be done, thus preventing any organ from being missed during exploration.[3] Adequate hydration of patient to prevent dehydration and blood reservation to check hypovolaemia prior to start of the surgery, the latter involving an unconventional manner of patient positioning due to impaled foreign body and a full length midline incision from xiphisternum to pubic symphysis significantly helps a thorough and safe wound exploration and repair of damaged structures.[3,7] Decision to take a CT of the abdomen is strictly based on the haemodynamic status, clinical and laboratory findings of the patient; as such it has a specificity of $97 \%$, accuracy of $82 \%$ and sensitivity rate of $64 \%$, but in unstable patients FAST (Focused Abdominal Sonography in Trauma) is more conducive.[7] A post-operative check CT scan can be done to confirm any residual injury.

\section{CONCLUSION}

The case Presented here stands as an Implausible Marvel due to the Features

1. Though multiple impalement injury is rare, double impalement at a common site is invariably far-fetched;

2. Despite such trauma, the patient had no signs of hypovolaemia;
3. Four structures barely escaped severe catastrophe, viz. right common iliac artery, right common iliac vein, right ureter, urinary bladder;

4. The small bowel was only grazed and though there was a tear in the mesentery, the bowel remained viable;

5. No signs of peritonitis despite contamination by two rusted iron rods;

6. Last but not the least- his miraculous post-operative uneventful recovery. The bowel segment though viable was resected and anastomosis done, so as to prevent any sepsis due to contaminated rusted iron rods [Figure 5]. This shows that prompt recognition of injury, timely and discriminative intervention without wastage on judicious resources and precise systematic care can prove beneficial in long-term survival of the patient.

\section{REFERENCES}

1. Badri Faisal, Al-Mazrouei Alya, Azam Hadeil, et al. Impalment injury-presentation of two new cases. Hamdan Medical Journal 2012;5(2):173-8.

2. Tantry Thrivikrama P, Kadam Dinesh, Shetty Pramal, et al. Penetrating abdominal injury in a polytrauma patient: anaesthetic challenges faced. J Anaesthesiol Clin Pharmacol 2011;27(2):272-4.

3. Kaur K, Singhal SK, Bhardwaj M, et al. Penetrating abdomino-thoracic injury with an iron rod: an anaesthetic challenge. Indian J Anaesth 2014;58(6):742-5.

4. Kolahdouzan Mohsen, Rezaee Mohammad T, Shahabi Shahab. Impalement thoracoabdominal trauma secondary to falling on metallic (Iron) bars: an extremely rare and unique case. Arch Trauma Res 2016;5(1):e18330.

5. Chih Hwang Nian, Ooi London Lucien. Acute surgical management. World scientific connecting great minds 2004;327-52.

6. Brooks A, Cotton B, Tai N, et.al. Emergency Surgery. Blackwell Publishing Ltd UK, 2010;139-91.

7. Schein Moshe, Rogers Paul. Schein's common sense emergency abdominal surgery. Springer-Verlag Berlin Heidelberg New York, 2005;297-304. 